\title{
Application and Analysis of Double Speed Loops in Planetary Gear Drive System
}

\author{
Sisi Chen ${ }^{1,2,3, a}$, Nanping Shao ${ }^{4, b}$, Xuanlin Huang ${ }^{1,2,3, c}$, Tao Tang ${ }^{1,2, d}$ \\ ${ }^{1}$ Key Laboratory of Optical Engineering,Chinese Academy of Sciences,Chengdu610209,China \\ 2Institute of Optics and Electronics,Chinese Academy of Science,Chengdu 610209,China \\ 3 University of Chinese Academy of Sciences,Beijing 100039,China \\ ${ }^{4}$ School of Automotive and Transportation,Xihua University,Chengdu 610039,China \\ achensisi13@yeah.net, bsnping000@foxmail.com, ${ }^{c} 138635416 @ q q . c o m l,{ }^{d}$ tangtao24@163.com \\ *Sisi Chen
}

Key words: double speed loop; backlash; planetary gear drive

\begin{abstract}
In order to reduce the influence of the backlash in the planetary gears, this paper presents a control method of the double speed loops. The motor speed loop is used as the inner loop and the load velocity loop as the outer loop. Combining with the theoretical analysis and simulation .It is concluded that the double speed loops has a good effect on the non - linear disturbance caused by the backlash.
\end{abstract}

\section{Introduction}

The current tracking control of the transmission mode torque motor direct drive and non-direct drive in two ways ${ }^{[1]}$, torque motor direct drive transmission accuracy ${ }^{[3]}$. Compared to the direct drive, non-direct drive in the planetary gear drive small size, high precision, small return gap, transmission ratio is stable, low power consumption, especially for small loads. It is widely used in aerospace, robotics, communications and other aspects ${ }^{[4]}$. However, although there are many advantages of planetary gear transmission, but the existence of backlash will inevitably increase the difficulty of control of the system, affecting the control accuracy of the system. In view of the non-linear problem of the backlash gap, scholars at home and abroad have done a lot of research ${ }^{[5]}$. Commonly used methods are mechanical backlash, multi-motor, inverse model compensation and other control methods. The mechanical method is by strengthening the gear rigidity, but this method can not ensure that no backlash problems are generated. In the literature [6], the double-motor drive is used to eliminate the backlash. The two motors have the effect of offsetting the offset voltage by applying the same voltage offset voltage to the two drive motors driving the same driven shaft. It is possible to avoid the uncontrollability of the single motor during the backlash, but the characterization is still a non-linear characteristic. Inverse model compensation is a scheme of reverse control, but because of the backlash nonlinear inverse model is not different and discontinuous, so prone to "jitter" phenomenon. In this paper, a control method based on double speed loops is adopted. The structure is simple and has excellent effect on the nonlinear disturbance such as the backlash of the planetary gear. The theoretical conditions are given to show that the double speed loops performance is better than the single speed loop performance. Finally, the simulation results show that the proposed control method is easy to implement and extremely effective.

\section{Planetary gear control system structure and performance analysis}

\subsection{Single speed loop control system structure and performance analysis}

Will only single-speed loop planetary gear drive model is simplified as shown in Figure 1: 


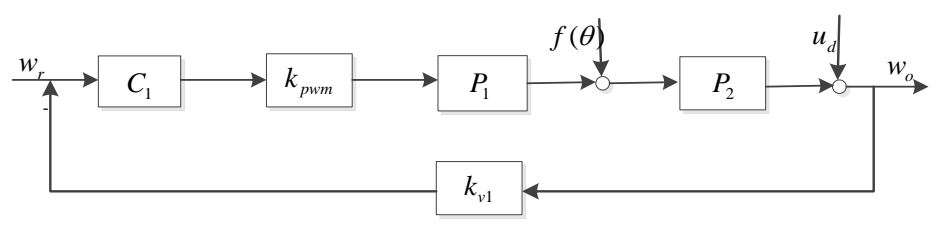

Fig.1. Planetary gear drive model for single speed loop

Which $w_{r}, w_{o}$ are the reference input angular velocity and the platform load output angular velocity, respectively, $f(\theta), u_{d}$ are planetary gear backlash and friction and other non-linear disturbances, $k_{v 1}$ is the scale factor for the speed component, $k_{p w m}$ is the magnification of the PWM power amplifier, $C_{1}, P_{1}, P_{2}$ are the load side speed loop controller, the mechanical transfer function of the motor and the transfer function of the load.

According to Fig. 1, we can derive the output angular velocity of the load $w_{o}$ relative to the friction disturbance torque $u_{d}$, the backlash of the nonlinear disturbance $f(\theta)$ and the parameter test input angular velocity $w_{r}$ transfer function is:

$$
w_{o}=\frac{P_{2} f(\theta)+u_{d}+k_{p w m} C_{1} P_{1} P_{2} w_{r}}{1+k_{v 1} k_{p w m} C_{1} P_{1} P_{2}}
$$

When designing a stable controller, it is guaranteed in the required frequency band:

$$
\left|k_{v 1} k_{p w m} C_{1} P_{1} P_{2}\right|>>1
$$

Then (1) can be approximated as:

$$
w_{o}=\frac{f(\theta)}{k_{v 1} k_{p w m} C_{1} P_{1}}+\frac{u_{d}}{k_{v 1} k_{p w m} C_{1} P_{1} P_{2}}+\frac{w_{r}}{k_{v 1}}
$$

It can be seen from (3) that in the single-speed ring planetary gear drive control system, the friction interference torque $u_{d}$, the backlash of the non-linear disturbance $f(\theta)$ on the load output angular velocity $w_{o}$ is done by the controller $C_{1}$, between the various disturbances mutual influence, so that the controller $C_{1}$ is difficult to coordinate, while the $u_{d}$ suppression is also related to the load $P_{2}$, so the use of this control loop can not be very good to suppress the disturbance and it is difficult to achieve high performance requirements.

\subsection{Two-speed loop control system structure and performance analysis}

Based on the advantages of cascade control and the analysis of single - speed loop control method, a method of double - speed loop control is proposed. In the control system design, the two-speed loop stability control is to measure the speed of the motor speed component of the inner loop, the use of another measurement element to measure the load speed loop constitutes the speed of the outer ring control. The speed inner ring includes the drive motor, which is mainly used to eliminate the influence of the non-linear characteristics of the backlash. The speed outer ring includes the load, which is mainly used for friction torque interference and external nonlinear disturbance. Speed outer ring controller and speed inner loop controller is usually added to the forward channel to form a two-speed loop control circuit, the control loop structure is shown in Figure 2: 


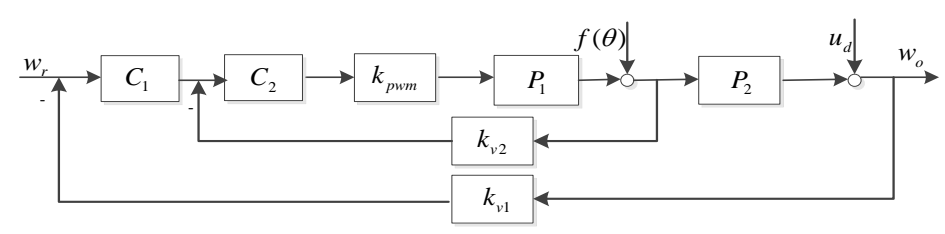

Fig. 2. Planetary gear drive model for two-speed loops

Which $w_{r}, w_{o}$ are the reference input angular velocity and the platform load output angular velocity, respectively, $f(\theta), u_{d}$ are planetary gear backlash and friction and other non-linear disturbances, $k_{v 1}$ is the scale factor for the speed component, $k_{p w m}$ is the magnification of the PWM power amplifier, $C_{1}, C_{2}, P_{1}, P_{2}$ are the load side speed loop controller, the motor speed loop controller, the mechanical transfer function of the motor and the transfer function of the load.

According to Fig. 2, we can derive the output angular velocity of the load $w_{o}$ relative to the friction disturbance torque $u_{d}$, the backlash of the nonlinear disturbance $f(\theta)$ and the parameter test input angular velocity $w_{r}$ transfer function is:

$$
w_{o}=\frac{k_{p w m} C_{1} C_{2} P_{1} P_{2} w_{r}+P_{2} f(\theta)+\left(1+k_{v 2} k_{p w m} C_{2} P_{1}\right) u_{d}}{1+k_{p w m} C_{2} P_{1}\left(k_{v 2}+k_{v 1} C_{1} P_{2}\right)}
$$

When designing a stable controller, it is guaranteed in the required frequency band:

$$
\begin{gathered}
\left|k_{p w m} C_{2} P_{1}\left(k_{v 2}+k_{v 1} C_{1} P_{2}\right)>>1\right| \\
\left|k_{v 2} k_{p w m} C_{2} P_{1}\right|>>1
\end{gathered}
$$

Then (4) can be approximated as:

$$
w_{o}=\frac{C_{1} P_{2} w_{r}}{k_{v 2}+k_{v 1} C_{1} P_{2}}+\frac{P_{2} f(\theta)}{k_{p w m} C_{2} P_{1}\left(k_{v 2}+k_{v 1} C_{1} P_{2}\right)}+\frac{k_{v 2} u_{d}}{k_{v 2}+k_{v 1} C_{1} P_{2}}
$$

It can be seen from (7) that in the two-speed loop stability control loop, the backlash suppression is mainly achieved by the speed of the inner loop controller, the speed of the outer loop controller from the auxiliary regulation; friction and other non-linear disturbance by the speed outer loop controller is determined, but has nothing to do with the change of system characteristic parameter, which is obviously improved compared with single speed loop control circuit.

For a control system with output $y$ under the given value $r$ and interference $d$, the system's anti-jamming capability can be measured by the signal-to-noise ratio $A_{i}=\frac{y / r}{y / d}$ to measure, if the molecules closer to the normal value, the denominator tends to 0 , then the anti-interference performance stronger ${ }^{[7][8]}$.

In the case of single-speed loop, the signal to noise ratio of the backlash and friction disturbance is:

$$
\begin{gathered}
A_{1 f}=k_{p w m} C_{1} P_{1} \\
A_{1 d}=k_{p w m} C_{1} P_{1} P_{2}
\end{gathered}
$$


Similarly, the signal-to-noise ratio of the double-speed loop to the backlash and friction disturbance is:

$$
\begin{gathered}
A_{2 f}=k_{p w m} C_{1} C_{2} P_{1} \\
A_{2 d}=\frac{k_{p w m} C_{1} C_{2} P_{1} P_{2}}{1+k_{v 2} k_{p w m} C_{2} P_{1}}
\end{gathered}
$$

In the design cascade control system, the order of the inner loop is generally low, so the $C_{2}$ gain coefficient can be made larger. Contrast (8) and (10), without loss of generality, when the controller uses proportional control, usually have $A_{2 f}>A_{1 f}$, So the double-speed loop has a stronger ability to overcome the backlash with respect to the single-speed loop control. For the friction and external nonlinear disturbance, the existence of the inner loop improves the dynamic characteristics of the controlled object and improves the stability The phase margin of the loop increases the open-loop gain and the anti-jamming capability is also improved with the single-speed loop control.

In addition, it can be seen from the system control structure diagram, in the single-speed loop system, the impact of the backlash need to go through the mechanical inertia of the lag in the load speed can be detected; and in the double speed loop system, the impact of the backlash can be detected by the speed sensor feedback on the motor speed detection and timely adjustment, improve the system response to interference speed effectively.

\section{Analysis of Single and Double Speed Loop Simulation Results}

In order to better analyze the influence of planetary gear transmission to the system, a system simulation model is established in Simulink module of Matlab. In the simulation of the planetary gear reduction ratio of 100, for a given sinusoidal signal, single speed loop system tracking results shown in Figure 3, double speed loops system tracking results shown in Figure 4.

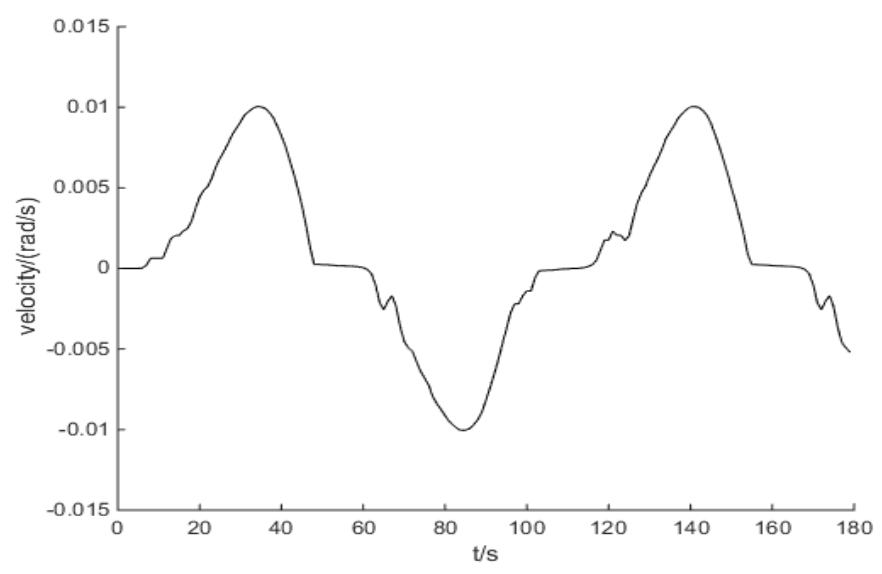

Fig. 3. Single-speed loop simulation results for planetary gear drives 


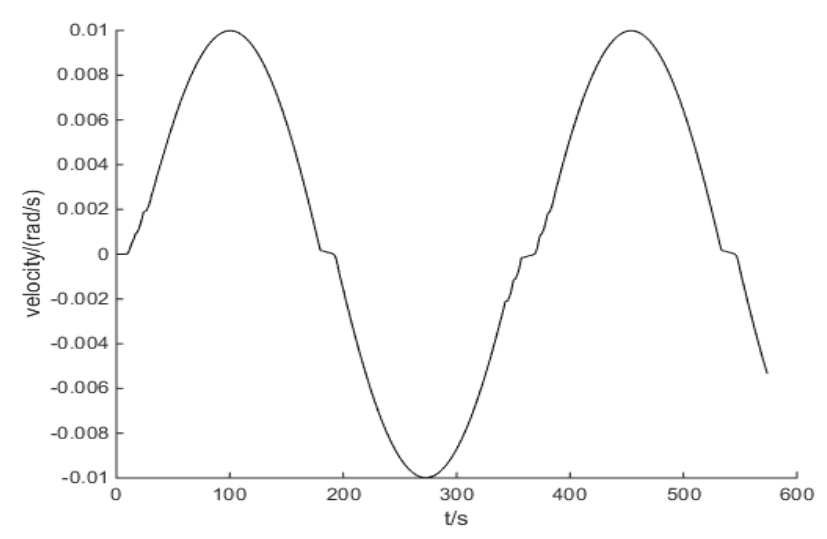

Fig. 4. Double speed loops simulation results for planetary gear drives

The results show that when the single-speed loop is adopted, there is a relatively large nonlinear dead band in the system, and the control structure of the two-speed loop can effectively suppress the non-linear characteristic in the system.

\section{Conclusions}

In this paper, a position control method based on two-speed loop is proposed, and the control performance of the system is analyzed. The control performance of the system is analyzed theoretically. The condition that the motor speed loop controller needs to be satisfied when the double speed loops performance is better than the single speed loop performance is obtained. Secondly, through the modeling and simulation of the planetary gear transmission model, the results show that for the planetary gear drive, the traditional single speed loop control structure is adopted, the system will have a larger dead zone, and the two speed loops can largely suppress the dead-zone characteristics present in the system.

\section{References}

[1] Huaxiang Cai,Disturbance compensation precision control techniques of tracking gimbal on telescope[D],pp.46-78,2016.(In Chinese)

[2] Huaxiang Cai, Yongmei Huang,Qiang Wang,Application and analysis of double speed loops in the harmonic drive system, Opto-electronic Engineering, vol.43, pp. 34-38, 2016.

[3] Hu Wang, Juan Chen,Lipeng Yin,Servo control technology in photoelectric tracking system, Journal of Changchun University of Technology, vol.30, pp. 533-539, 2009.(In Chinese)

[4] Maxon, High precision drive and systems, Maxon Motor, vol. 2 pp. 67-100, 2014.

[5] Qingwei Chen,Yu Guo,Jingzhong Yang,Application of improving the precision of backlash nonlinear system ,Journal of Nanjing University of Science and Technology, vol. 27, pp. 127-132, 2007.(In Chinese)

[6] Guofeng Zhao,Qingwei Chen, Back lit nonlinear adaptive control of dual motor drive servo system, Journal of Nanjing University of Science and Technology, vol. 31, pp. 187-192, 2008.

[7] Yongmei Huang,Jiaguang Ma,Chengyu Fu, Application simulation of predictive filtering technology in photoelectric theodolite, Opto-electronic Engineering, vol. 29, pp. 5-9, 2002.

[8]Michel Verhaegen, Paul, Van Dooren. Numerical Aspects of Different Kalman Filter.,IEEE Transaction on Automatic Control,vol.31, pp.907-917, 1986. 